\title{
Developmental Adaptations in Cytosolic Phosphate Content and pH Regulation in the Sheep Heart In Vivo
}

\author{
Michael A. Portman* and Xue-Han Ning ${ }^{\ddagger}$ \\ With the technical assistance of Thomas G. Cooper ${ }^{5}$ and Deborah Simon* \\ Departments of Pediatrics and Human Development, ${ }^{*}$ Physiology, ${ }^{\ddagger}$ and Radiology, ${ }^{\S}$ College of Human Medicine, \\ Michigan State University, East Lansing, Michigan 48824
}

\begin{abstract}
This study examines adaptations in myocardial cytosolic phosphate content and buffering capacity that occur in vivo as a function of development. Phosphate metabolites were monitored in an open chest sheep preparation using a ${ }^{31} \mathbf{P}$ magnetic resonance surface coil over the left ventricle. Newborn lambs (aged 4-9 d, $n=5$ ) underwent exchange transfusion with adult blood to reduce blood-borne 2,3-diphosphoglycerate contamination of the heart monophosphate and phosphomonoester resonances, thus allowing determination of these phosphate concentrations. The blood-exchanged newborns and mature controls (aged 30-60 d, $n=5$ ) were infused with $0.4 \mathrm{~N}$ hydrochloric acid to decrease $\mathrm{pH}$ from $>7.35$ to $<7.00$. Simultaneously, intracellular and extracellular pH were determined from the chemical shifts of the respective phosphate peaks and compared to arterial blood $\mathrm{pH}$. Findings were as follows: (a) diphosphoglycerate contribution to the cardiac spectrum was found to be negligible, (b) significant decreases in cytosolic phosphate $(P<0.03)$ and phosphomonoester $(P<0.01)$ content occurred with maturation, and $(c)$ large decreases in extracellular pH ( $>0.5 \mathrm{U})$ in both groups were similarly associated with only small changes in intracellular $\mathrm{pH}(<0.1 \mathrm{U})$. Change in cytosolic phosphate content implies that alterations occur in the phosphorylation potential with resulting effects on regulation of myocardial respiration, and cardiac energetics. (J. Clin. Invest. 1990. 86:1823-1828.) Key words: heart • magnetic resonance spectroscopy $\bullet \mathrm{pH} \bullet$ phosphate
\end{abstract}

\section{Introduction}

The relation between myocardial phosphate metabolites and oxygen consumption changes as a function of development (1). Cytosolic levels of the ATP hydrolysis products, inorganic monophosphate $\left(\mathrm{P}_{\mathrm{i}}\right)$, and ADP directly increase with myocardial oxygen consumption in the neonatal heart in vivo. These metabolite concentrations remain stable in mature myocardium during similar work increases (1-4). Therefore, regulation of oxidative phosphorylation in the neonatal heart in vivo appears to depend more on mitochondrial feedback via the ATP hydrolysis products. The degree of feedback control,

Address reprint requests to Dr. Portman, Department of Pediatrics and Human Development, B401 Clinical Center, Michigan State University, East Lansing, MI 48824.

Received for publication 21 March 1990 and in revised form 15 June 1990.

J. Clin. Invest.

(C) The American Society for Clinical Investigation, Inc. 0021-9738/90/12/1823/06 \$2.00

Volume 86, December 1990, 1823-1828 however, remains unknown, because accurate calculation of myocardial $P_{i}$ levels in the newborn has not been performed in vivo. For similar reasons, the role that cytosolic $\mathrm{pH}$ plays in the regulation of myocardial energy metabolism also remains undefined.

${ }^{31}$ Phosphorous nuclear magnetic resonance spectroscopy $\left({ }^{31} \mathrm{P} N M R\right)^{1}$ is now the primary tool for studying these relations in vivo (1-5). NMR yields dynamic measurements of intracellular phosphate and avoids overestimation errors associated with freeze clamp and acid extraction techniques (6). Furthermore, intracellular $\mathrm{pH}$ can be calculated concomitantly from the chemical shift of the monophosphate resonance peak $(7,8)$. NMR analysis of myocardial intracellular monophosphate levels and $\mathrm{pH}$ has been complicated by the contamination of the $P_{i}$ resonance peaks by phosphomonoesters, particularly 2,3-diphosphoglycerate (2,3-DPG), present in blood perfusing the myocardium or within the ventricular chamber (5). This problem has been circumvented in mature myocardium by studying sheep $(1,9)$, a species in which blood 2,3-DPG levels drop dramatically after the first week of life (10). However, possible 2,3-DPG contamination in neonatal sheep heart spectra has hampered calculation of intracellular $P_{i}$ concentrations and $\mathrm{pH}$ in the newborn heart $(1,11)$. Therefore, the purpose of this study was to determine intracellular phosphate and phosphomonoester levels, and the buffering capability of the newborn myocardium in vivo. This was accomplished by exchange-transfusing neonatal lambs with adult sheep blood in order to reduce contamination of the phosphate peaks by blood-borne 2,3-DPG. The $P_{i}$ peak resolution and chemical shift data obtained in these experiments were then used to assess buffering capability by examining the response of neonatal myocardial $\mathrm{pH}$ to extracellular acidosis.

\section{Methods}

Animal preparation. Two age groups of mixed breed Western sheep were used for this study. Neonatal lambs ranged between 3 and $9 \mathrm{~d}$ of age (mean $5.6 \pm 1.39 \mathrm{~d}, n=5$ ), and weighed between 4 and $6 \mathrm{~kg}$. The size of mature sheep used in this study was dictated by the magnet's 26-cm clear bore, which precluded the use of sheep larger than $20 \mathrm{~kg}$. Consequently, sheep between 30 and $60 \mathrm{~d}$ of age (mean $47 \pm 6.8 \mathrm{~d}, n$ $=5$ ) were used.

Animals were sedated with an intramuscular injection of $10 \mathrm{mg} / \mathrm{kg}$ ketamine and $0.2-0.4 \mathrm{mg} / \mathrm{kg}$ xylazine, intubated, and then ventilated (model D-900 pediatric ventilator, Siemens-ELEma, Schaumberg, IL) with an anesthesia mixture of $0.5-1.0 \%$ halothane and $\sim 99 \%$ oxygen. Femoral arterial cannulation was performed for monitoring systemic blood pressure and sampling blood. A cannula was inserted in each jugular vein for intravenous infusions and blood exchange. Arterial pH was maintained between 7.35 and 7.45 by adjustment of ventilatory

1. Abbreviations used in this paper: 2,3-DPG, 2,3-diphosphoglycerate; NMR, nuclear magnetic resonance; $\mathrm{PCr}$, phosphocreatine. 
tidal volume and correction of metabolic acidosis with sodium bicarbonate. After a median sternotomy, the pericardial fat pad was exposed and removed. Platinum-tipped pacing electrodes were sutured to the right atrial appendage. An ellipsoid-shaped NMR surface coil, which conformed to the general shape of the sheep heart was sutured to the pericardium overlying the left ventricle. This coil placement allowed unrestricted cardiac movement and filling, while keeping the coil in reproducible proximity of the heart. The thoracotomy opening was then sealed with plastic wrap to prevent water loss. The lamb was wrapped in a water-circulating heating blanket that maintained body core temperature at $\sim 38^{\circ} \mathrm{C}$, and placed in a lucite cradle which fit into the 26-cm clear bore of the 4.7-T magnet. The surface coil was positioned at the magnetic center of the system.

$N M R$ measurements. Upon transfer of the lamb into the magnet, the NMR surface coil was tuned to $81 \mathrm{MHz}$ and matched to $50 \Omega$ by using a network analyzer with a connecting cable calibrated for use within the magnet field. Respiration was maintained within the magnet via 15 feet of tygon tubing attached to the expiratory and inspiratory ports of the ventilator. Blood pressure was monitored using a solid-state nonmagnetic pressure transducer (Cobe Laboratory Inc., Lakewood, $\mathrm{CO}$ ) positioned just outside the magnet bore.

NMR data were collected with a chemical shift imaging spectrometer (General Electric Co., Fremont, CA) employing OMEGA software (General Electric Co.). Shimming on the proton-free induction decay at $200 \mathrm{MHz}$ and acquisition of ${ }^{31} \mathrm{P}$ spectra were performed as previously described (9). Cardiac and respiratory gating were performed in all studies using a personal computer outfitted with a data acquisition board and software (Labtech, Burlington, MA). The computer triggered the spectrometer, the begin inspiratory input on the ventilator, and the cardiac stimulator simultaneously, using a basic harmonic of $30 \mathrm{cycles} / \mathrm{min}$. This yielded an interpulse delay of $2 \mathrm{~s}$. Cardiac pacing was performed at a frequency which was the lowest harmonic of the respiratory rate, above the animal's intrinsic heart rate. This gating method minimized variation in cardiac position during multiple acquisitions and improved magnetic field shimming around the heart. Before each experiment, the radio frequency pulse width necessary to obtain the maximum phosphocreatine ( $\mathrm{PCr})^{31} \mathrm{P}$ NMR signal from the heart was determined. Using a $100-\mathrm{W}$ amplifier this varied from 30 to $45 \mu$ s according to heart size.

Correction for saturation was performed by utilizing a 16-s interpulse delay in five animals to obtain fully relaxed spectra. Comparison of fully relaxed spectra with those obtained with a 2-s interpulse delay yielded the necessary ratio to perform correction for differential saturation of the phosphate peaks.

Transfusion protocol. Before initiation of the exchange, $10 \mathrm{~min}$ of baseline ${ }^{31} \mathrm{P}$ NMR and hemodynamic data was obtained. Additionally, blood samples were obtained for determination of arterial $\mathrm{pH}, \mathrm{PO}_{2}$, $\mathrm{PCO}_{2}$, hemoglobin, 2,3-DPG, and $\mathrm{P}_{\mathrm{i}}$ content. Exchange was begun by transfusing $25-30 \mathrm{ml}$ of citrated fresh adult sheep blood, followed by simultaneous withdrawal and transfusion of $100-150 \mathrm{ml} / \mathrm{kg}$ for $\sim 1 \mathrm{~h}$. NMR data were obtained in 10-min acquisition blocks (300 scans) throughout the procedure. Arterial blood pressure and blood gasses were closely monitored throughout the study, and the procedure was slowed if there was $>10 \%$ decrease in systolic pressure. In general, blood pressure was stable throughout these experiments. After the exchange $20 \mathrm{~min}$ was allowed for equilibration, after which blood samples and NMR acquisition were repeated.

Induction of metabolic acidosis. After completion of the transfusion protocol in newborn lambs, $0.4 \mathrm{~N} \mathrm{HCl}$ was infused at a rate of 1-2 $\mathrm{ml} / \mathrm{min}$. Data was obtained in 10-min blocks as described above. Arterial $\mathrm{pH}$ was closely monitored and experiments were terminated once a $\mathrm{pH}$ of 6.9 was attained. This usually took $1-1 \frac{1}{2} \mathrm{~h}$. Mature lambs also underwent acid infusion but not exchange transfusion since their 2,3 DPG levels were already low.

Blood phosphate metabolites. Blood 2,3-DPG and $\mathbf{P}_{\mathrm{i}}$ concentrations were determined using standard spectrophotometric techniques at $340 \mathrm{~nm}$ procedures $35 \mathrm{UV}$ and $360 \mathrm{UV}$ (Sigma Diagnostics, St. Louis, MO).
Data analysis. Resulting spectra were analyzed and peak areas were determined with an automated Lorentzian line fitting program employing peak deconvolution, which was specially written for the General Electric-OMEGA system. All NMR peak area data were corrected for differential saturation. Extracellular and intracellular $\mathrm{pH}$ were determined by measurement of the chemical shift of the intra- and extracellular phosphate peaks with reference to $\mathrm{PCr}$. The $\mathrm{pH}$ was then determined from a previously derived calibration curve constructed using a solution of $50 \mathrm{mM} \mathrm{KCl}, 30 \mathrm{mM} \mathrm{NaCl}, 1 \mathrm{mM} \mathrm{MgSO}_{4}, 1 \mu \mathrm{M}$ $\mathrm{CaCl}, 1 \mathrm{mM} \mathrm{PCr}$, and $2 \mathrm{mM} \mathrm{NaPO}{ }_{4}$ at $37^{\circ} \mathrm{C}$ in which the chemical shift difference between $\mathrm{P}_{\mathrm{i}}$ and $\mathrm{PCr}$ was determined as a function of pH (5).

Pre- and postexchange data were subjected to a two-tailed paired $t$ test, as were data before and at completion of the acid infusion protocol. Comparisons between mature and newborn sheep utilized unpaired $t$ tests. The relations of intracellular and extracellular $\mathrm{pH}$ were analyzed with linear regression. A $t$ test was used to compare the slopes of the lines obtained from newborn and mature sheep data.

\section{Results}

NMR spectra resolution. Spectra from a newborn and mature heart are shown in Fig. 1. Proton line widths ranging between 32 and $54 \mathrm{~Hz}$ were achieved through shimming in all experiments included for analysis in this study. This resolution enabled the curve fitting program to separate and analyze the
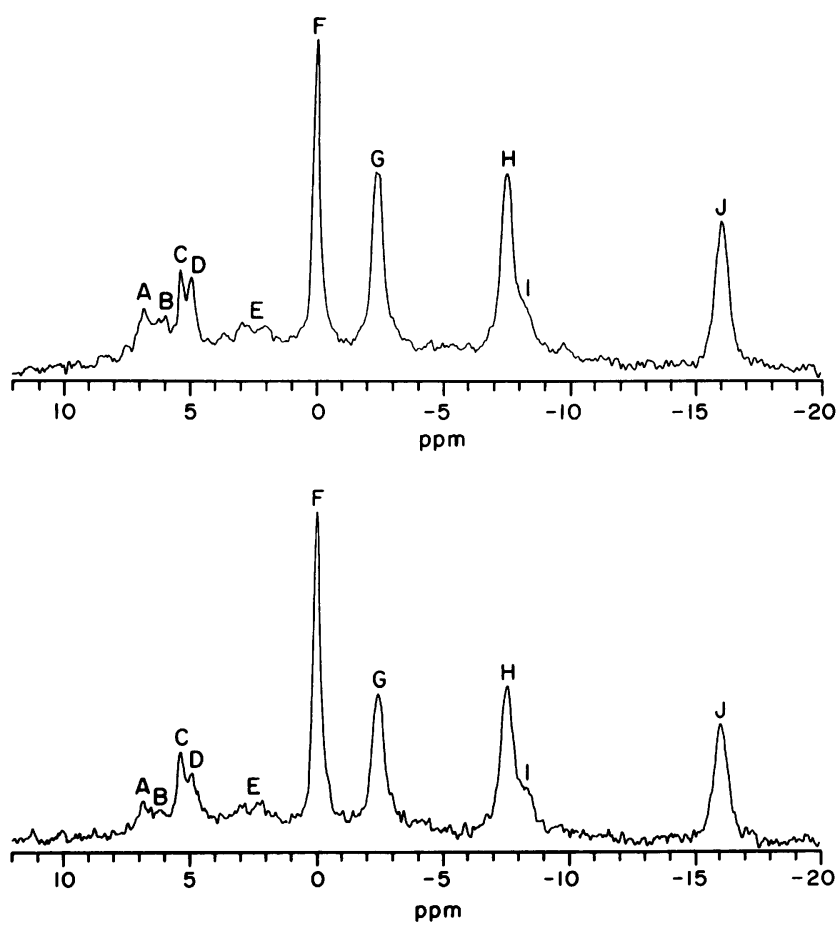

Figure 1. $(A){ }^{31} \mathrm{P}$ NMR spectrum from a newborn lamb left ventricle (aged $4 \mathrm{~d}$ ) in vivo. The spectrum represents the sum of 300 acquisitions obtained with a 2-s interpulse delay. $5-\mathrm{Hz}$ line broadening was applied before Fourier transformation. Peak assignments are phosphomonoesters ( $A$ at $6.80 \mathrm{ppm}$ and $B$ at $6.10 \mathrm{ppm}$ ), extracellular $P_{\mathrm{i}}$ ( $C$ at $5.25 \mathrm{ppm}$ ), intracellular $\mathrm{P}_{\mathrm{i}}(D$ at $4.90 \mathrm{ppm})$, phosphodiesters $(E$ at $2.0-3.0 \mathrm{ppm}), \operatorname{PCr}(F$ at $0.0 \mathrm{ppm}), \gamma-\mathrm{ATP}(G$ at $-2.50 \mathrm{ppm})$, $\alpha-\operatorname{ATP}(H$ at $-7.55 \mathrm{ppm}), \operatorname{NAD}(I$ at $-8.30 \mathrm{ppm})$, and $\beta$-ATP ( $J$ at $16.04 \mathrm{ppm})$. (B) Spectrum similarly obtained and processed from a 60 -d-old sheep. Peak assignments are similar to those in $A$. Note that there is a decrease in phosphomonoester and intracellular $P_{i}$ peak areas, when compared to $A$. 

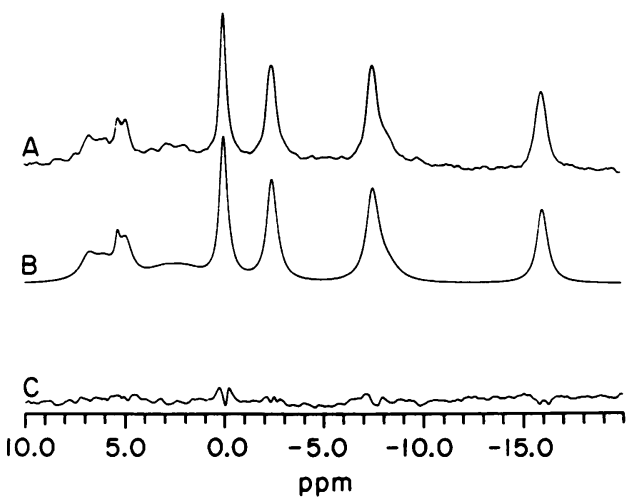

Figure 2. Illustration of Lorentzian line fitting utilizing a peak deconvolution program. Trace $A$ is the Fourier transformed spectrum for a lamb heart; trace $B$ is the spectrum generated from the computed fit; trace $C$ is the difference between the two $(B-A)$.

individual peaks of the complex phosphomonoester- $\mathrm{P}_{\mathrm{i}}$ region (Fig. 2). PCr signal-to-noise ratio was $>100: 1$ with employment of only $5 \mathrm{~Hz}$ exponential multiplication.

Exchange transfusion. Data are summarized in Tables I and II. Lambs were hemodynamically stable throughout the exchange with minimal or no change in blood pressure and/or pacing rate as illustrated in Table I. Additionally, pre- and postexchange $\mathrm{PCr}$ and ATP content of the left ventricular wall were essentially the same. The maximum blood 2,3-DPG concentration $(1.96 \mathrm{mM})$ was noted in a 4-d-old lamb. Despite substantial reductions in 2,3-DPG in all animals, there were no significant changes in phosphomonoester/ATP or $\mathrm{P}_{\mathrm{i}} / \mathrm{ATP}$. Blood phosphate levels (Table II) changed minimally through the transfusion. This was consistent with the stability in the extracellular monophosphate peak in the ${ }^{31} \mathrm{P}$ NMR spectrum. Difference spectra of the expanded monophosphate region also demonstrated this consistency (Fig. 3).

Control physiologic parameters and relative concentrations of ${ }^{31} P$ metabolites. The mean control values for both age groups and results of the unpaired $t$ test are summarized in Table I. Statistical significance was defined at the $95 \%$ confidence level. Because there was no significant difference in relative phosphate concentrations between pre- and postexchange transfusion data from the newborn lambs, the latter was used for comparison in the table. Preexchange data was also com- pared with mature control data and yielded the same results with regards to significance. Significant differences in the phosphomonoester/ATP and $\mathrm{P}_{\mathrm{i}} / \mathrm{ATP}$ between the newborn and mature myocardium were noted while $\mathrm{PCr} / \mathrm{ATP}$ values were similar. These differences are made obvious by examination of typical newborn (Fig. 1) and mature heart spectra and the knowledge that 2,3-DPG contribution to those peaks is insignificant. Using an ATP concentration of $8.6 \mathrm{mM}$ obtained from previously published freeze clamp studies (1), intracellular $P_{i}$ concentration is estimated from $P_{i} / A T P$ at 3.7 $\mathrm{mM}$ in the newborn myocardium and $2.3 \mathrm{mM}$ in mature.

Response to metabolic acidosis. A summary of the hemodynamic response to acid infusion is provided in Table $\mathrm{I}$. The pacing rate was always kept at a harmonic of 30 beats $/ \mathrm{min}$. Therefore, if the intrinsic heart rate rose and competed with pacing, then the pacing rate was increased to the next harmonic. Spectra from an acid infusion experiment are illustrated in Fig. 4. The accuracy of the NMR method for measuring $\mathrm{pH}$ was assessed by comparing the arterial $\mathrm{pH}$ with extracellular $\mathrm{pH}$ as determined from the chemical shift of extracellular monophosphate (Fig. 5). Linear regression analysis using calculated extracellular $\mathrm{pH}$ as the dependent variable yielded a slope of $0.99 \pm 0.046$, and $r=0.95$. Baseline intracellular $\mathrm{pH}$ was $7.13 \pm 0.03$ for exchanged newborns, and $7.08 \pm 0.02$ for mature sheep. The relations between arterial $\mathrm{pH}$ and $\mathrm{pH}_{\mathrm{i}}$ are shown in Fig. 6. Substantial changes in arterial $\mathrm{pH}$ $(>0.5 \mathrm{pH} \mathrm{U})$ were accompanied by only $0.1 \mathrm{pH}_{\mathrm{i}}$ change in both groups of animals. There was no significant difference in the $\mathrm{pH}_{\mathrm{i}}$ response between newborn and mature myocardium as determined by $t$ test comparison of the regression line slopes.

\section{Discussion}

The 2,3-DPG contribution to the myocardial phosphate spectrum is mainly dependent on three factors: $(a)$ the blood 2,3DPG concentration, $(b)$ the percentage of myocardium which is vascular, and $(c)$ the depth of radiofrequency signal penetration into the blood-filled ventricle. This study indicates that the 2,3-DPG contribution to the phosphomonoester and monophosphate peaks is negligible in newborn lamb hearts, thus allowing analysis of intracellular myocardial content of these metabolites. These results are comparable to oxypherol blood replacement studies performed in canines, which

Table I. Hemodynamic and ${ }^{31} P$ Parameters

\begin{tabular}{|c|c|c|c|c|c|c|}
\hline & \multicolumn{3}{|c|}{ Newborn (3-9 d) } & \multicolumn{2}{|c|}{ Mature (39-70 d) } & $t$ \\
\hline Mean arterial pressure $(\mathrm{mm} \mathrm{Hg})$ & $67 \pm 9.7$ & $67 \pm 10.9$ & $66 \pm 6.7$ & $72 \pm 3.7$ & $69 \pm 4.6$ & NS \\
\hline PCr/ATP & $1.69 \pm 0.086$ & $1.74 \pm 0.062$ & $1.69 \pm 0.086$ & $1.93 \pm 0.113$ & $1.83 \pm 0.141$ & NS \\
\hline Phosphomonoester/ATP & $0.66 \pm 0.068$ & $0.68 \pm 0.097$ & $0.66 \pm 0.011$ & $0.26 \pm 0.028$ & $0.33 \pm 0.051$ & $P<0.01$ \\
\hline
\end{tabular}

Data are presented in columns from the baseline, postexchange transfusion, and final acidosis periods for newborns; baseline and final acidosis periods for mature sheep. Unpaired $t$ test results are presented in the last column. PCr/ATP, phosphomonoester/ATP, $\mathrm{P}_{\mathrm{i}} / \mathrm{ATP}$ are the respective ratios of the areas of phosphocreatine, phosphomonoesters, and intracellular phosphate peaks to the $\beta$-ATP peak. ND indicates that no measurement was done, owing to convergence of the extracellular and intracellular phosphate peaks during acidosis. ${ }^{*}$ Column contains parameters for which unpaired $t$ tests were performed. 
Table II. Blood 2,3-DPG and Phosphate Levels

\begin{tabular}{lllc}
\hline & \multicolumn{3}{c}{ Newborn } \\
\cline { 2 - 3 } & \multicolumn{1}{c}{ Pre } & \multicolumn{1}{c}{ Post } & Mature \\
\hline & & $m M / l i t e r$ & \\
DPG & $1.84 \pm 0.060$ & $0.45 \pm 0.156$ & $<0.02$ \\
Phosphate & $9.65 \pm 0.42$ & $8.52 \pm 0.64$ & $5.90 \pm 0.34$ \\
\hline
\end{tabular}

Blood 2,3-DPG and $P_{i}$ concentrations are presented for newborns, pre- and postexchange transfusion, and for mature sheep at baseline.

showed that 2,3-DPG signal was $\sim 10 \%$ of the ATP signal using similar methods (5). However, newborn lamb blood 2,3DPG levels are substantially lower than in canines, thereby further reducing its possible contribution to the $P_{i}$ signal. Radiofrequency penetration has been shown to be roughly equivalent to the surface coil radius (5), which was $1 \mathrm{~cm}$ for these studies. Since the left ventricular wall of the newborn lamb is usually $>0.8 \mathrm{~cm}$, and the coil is positioned over the apex where the ventricular volume is small, 2,3-DPG signal contribution from blood in the ventricular chamber would be minimal. Establishing that the observed signal is strictly from myocardium, and not from blood-borne 2,3-DPG, enables detailed analysis of the myocardial phosphorous spectrum.

Free intracellular phosphate levels reported in this study are substantially higher than those found in mature canine
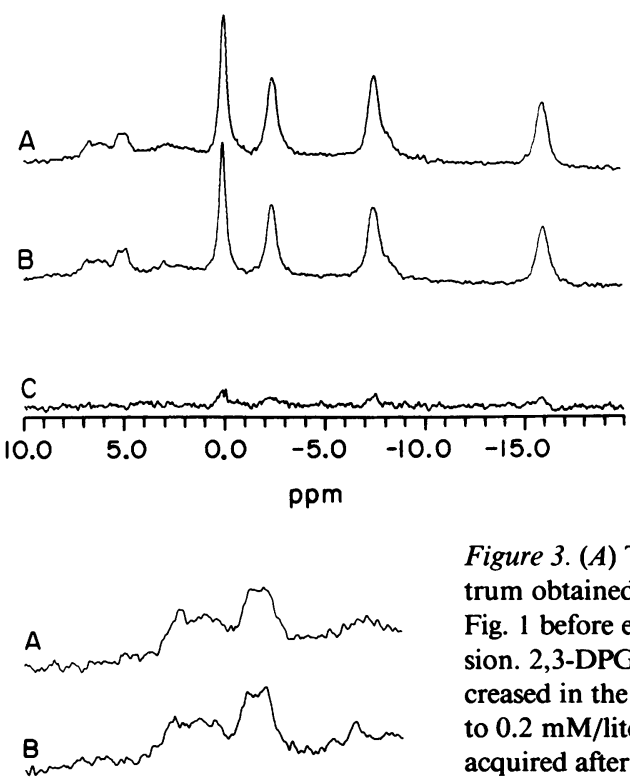

Figure 3. $(A)$ Trace $A$ is a spectrum obtained as described in Fig. 1 before exchange transfusion. 2,3-DPG was then decreased in the blood from 1.9 to $0.2 \mathrm{mM}$ /liter. Trace $B$ was acquired after completion of the exchange. Trace $C$ is the difference between the preand postexchange spectra $(B$ small increase in PCr and ATP
smans

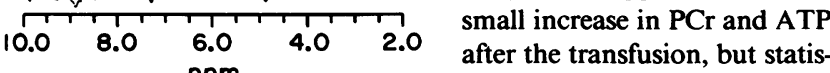
ppm tical analysis of all data shows

this to be insignificant. Additionally, no concomitant decrease in intracellular phosphate occurs. (B) $a$ magnification of $A$ from the phosphomonoester- $P_{i}$ region of the spectrum. No noticeable difference in the phosphomonoester or $P_{i}$ peaks accompanies the dramatic decrease in blood 2,3-DPG concentration.

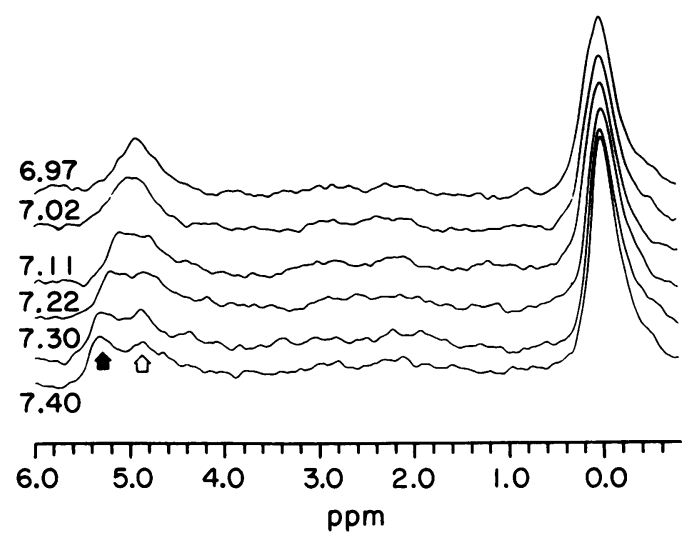

Figure 4. A stackplot of successive spectra obtained during an acid infusion experiment. The average arterial $\mathrm{pH}$ during each spectrum's acquisition is noted at the left. The chemical shift of the intracellular $\mathbf{P}_{i}$ peak (open arrow) stays relatively constant as the extracellular $\mathbf{P}_{i}$ peak (solid arrow) reflects decreasing $\mathrm{pH}$ and shifts towards $\mathrm{PCr}$ (at $0.0 \mathrm{ppm}$ ) until the two peaks converge at $\mathrm{pH}=6.97$.

myocardium (950 $\mu \mathrm{M} /$ liter), using similar methods (5). This difference is likely due to interspecies variability in total phosphate content, as even blood phosphorous levels are substantially higher in sheep (12). Additionally, levels in mature sheep were slightly lower in the first author's previous work. This discrepancy is attributed to the improved resolution and signal-to-noise ratio obtained in the present study. A small but significant decrease in intracellular phosphate concentration was noted to occur with maturation, although $\mathrm{PCr}$ and ATP concentrations remained stable. The ramifications of this developmental alteration can be reviewed in terms of both mitochondrial regulation and cardiac energetics. The classic Michaelis-Menton kinetic model of myocardial respiratory control, which is based on in vitro data from isolated mitochondria, postulates that feedback regulation of oxidative phosphorylation occurs via changes in the ATP hydrolysis products, ADP and $P_{i}(2)$. However, the calculated phosphate $K_{\mathrm{m}}$ of $900 \mu \mathrm{M}$ generally used in that model is well below the in

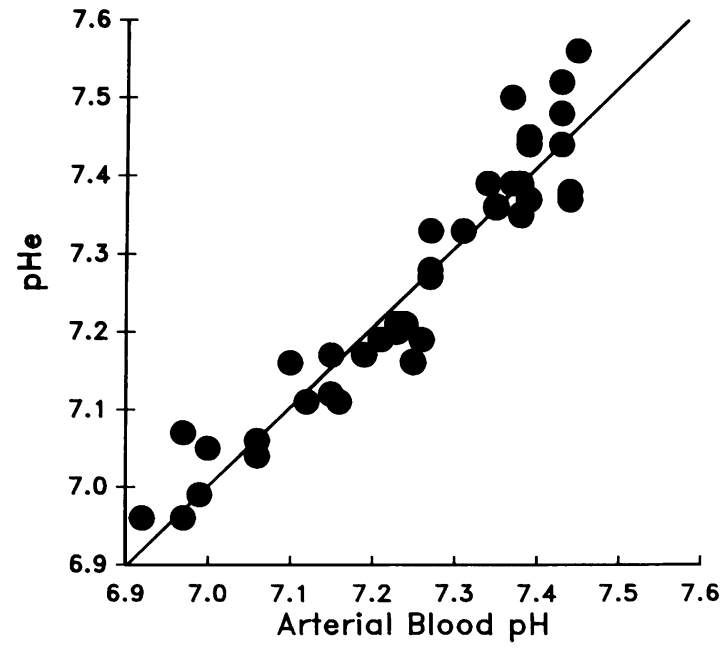

Figure 5. Extracellular $\mathrm{pH}\left(\mathrm{pH}_{\mathrm{e}}\right)$ as determined by the NMR chemical shift of extracellular $\mathrm{P}_{\mathrm{i}}$ is plotted against the arterial $\mathrm{pH}$. Linear regression analysis yields a slope of $0.99 \pm 0.46(\mathrm{SE})$, and $r=0.95$. 

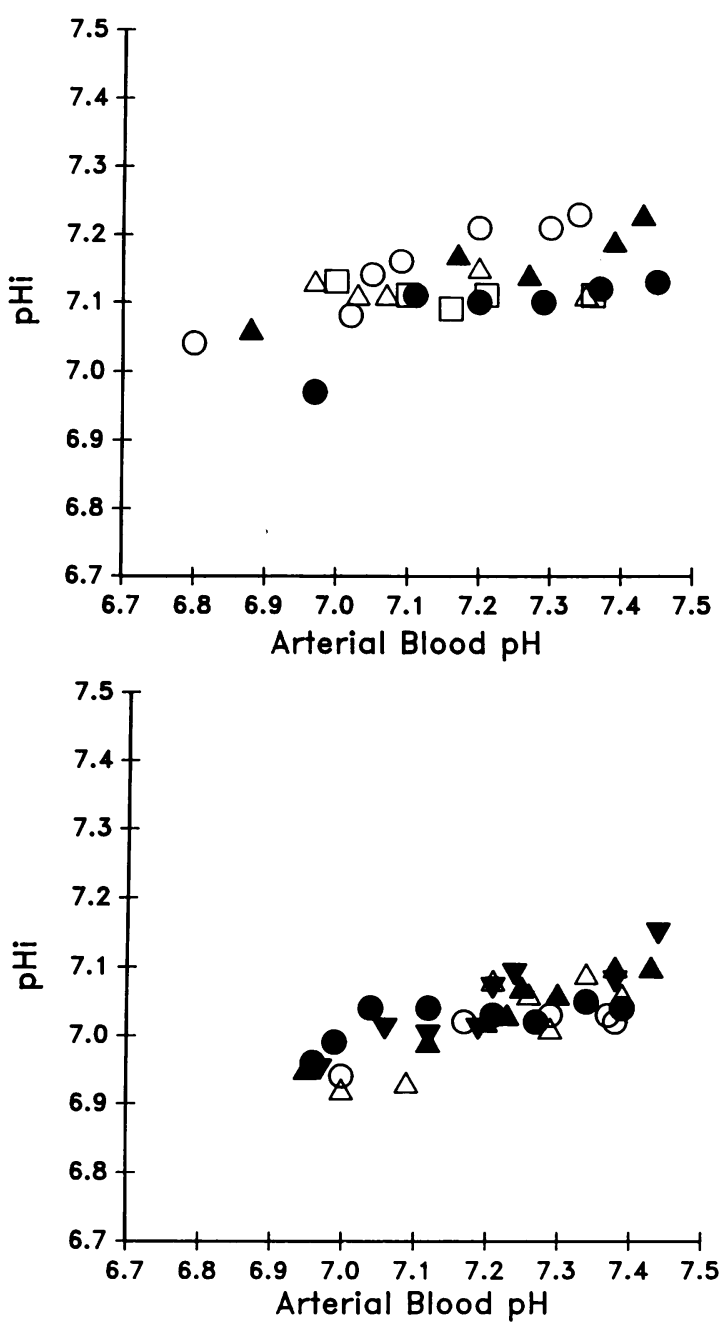

Figure 6. Myocardial intracellular $\mathrm{pH}\left(\mathrm{pH}_{\mathrm{i}}\right)$ is plotted vs. arterial $\mathrm{pH}$ for acid infusions in $(A)$ newborn and $(B)$ mature sheep. Each symbol represents a different animal. Linear regression analysis yields a slopes of $0.196 \pm 0.05$ for newborns and $0.194 \pm 0.05$ for mature, indicating that the relationship between extracellular and intracellular $\mathrm{pH}$ is similar in the two groups.

vivo cytosolic phosphate concentration determined in this study and would thereby make myocardial oxygen consumption relatively insensitive to $P_{i}$ changes. Nevertheless, a previous study performed in newborn lambs estimated that $P_{i}$ increased with myocardial oxygen consumption in magnitude near that predicted by isolated mitochondria models (1). However, because the 2,3-DPG contamination was considered substantial, low and inaccurate baseline levels of $P_{i}(1.6 \mathrm{mM} /$ liter) were assumed for those estimations. Recalculation based on the higher concentrations found in this study, shows that although $P_{i}$ change varies directly with work in the newborn heart, the degree is much less than expected in the MichaelisMenton model. The presence of higher intracellular $P_{i}$ levels also indicates that the newborn myocardium functions at a lower phosphorylation potential [ATP]/[ADP][ $\left.\mathrm{P}_{\mathrm{i}}\right]$, and thus a lower free energy change for ATP synthesis. The free energy change would be driven lower at higher work levels as both $P_{i}$ and ADP increase. Whether or not this thermodynamic alteration has any functional impact on the newborn heart is purely speculative.
An additional finding in this study is that the phosphomonoester concentration in newborn myocardium is considerably higher than in mature. Two major phosphomonoester peaks occur consistently at 6.86 and $6.00 \mathrm{ppm}$. Detailed analysis has shown that these chemical shifts are consistent with those of phosphorylethanolamine and phosphorylcholine at physiologic $\mathrm{pH}(13,14)$. Elevated concentrations of these phosphomonoesters, which are membrane phospholipid precursors, have also been noted to occur in other developing tissues including canine (13) and rat brain (15), and rat skeletal muscle (16). It has been theorized that high phosphomonoester concentrations may stimulate membrane synthesis (17). This could explain their prominence in developing myocardium that is undergoing rapid hypertrophy.

Resolution of the extracellular from the intracellular phosphate peak in this study enables determination of $P_{i}$ chemical shift and thus the $\mathrm{pH}$ in each respective compartment. The relation of the NMR determined extracellular $\mathrm{pH}$ with arterial $\mathrm{pH}$ is illustrated in Fig. 5. The strong correlation with minimal scatter in this plot indicates that arterial $\mathrm{pH}$ is similar to extracellular $\mathrm{pH}$ as derived from the ${ }^{31} \mathrm{P}$ chemical shift. The extracellular phosphate peak must contain a vascular and an interstitial component, but the proportional contribution is impossible to determine. However, the narrow line width of the extracellular phosphate peak indicates that the $\mathrm{pH}$ is relatively homogenous throughout the extracellular space in this aerobic model. Extracellular $\mathrm{pH}$ measurement was limited only at very low arterial $\mathrm{pH}$ where the extracellular phosphate peak shifted and merged with the intracellular $P_{i}$ peak as the two $\mathrm{pH}$ values equilibrated. Even so, the convergence of the two peaks (Fig. 4) illustrates that extracellular $\mathrm{pH}$ falls much more rapidly than intracellular.

The role that intracellular hydrogen ion concentration may have in influencing myocardial metabolism in vivo has been a subject of considerable controversy. This stems partially from methodological problems involving $\mathrm{pH}$ electrode studies, which require meticulous impalement of the cell to achieve an accurate reading of intracellular $\mathrm{pH}$ (18). Errors in impalement or severe disruption of cell membranes may yield "tissue" $\mathrm{pH}$ readings which are actually from a hybrid of interstitial and intracellular environment. This results in higher intracellular $\mathrm{pH}$ readings than found during nuclear magnetic resonance studies $(5,19,20)$, as well as the assumption that myocardial intracellular $\mathrm{pH}$ is closely related to tissue $(19,20)$. Results of in vivo pH electrode studies generally show that intracellular $\mathrm{pH}$ changes in magnitude similar to extracellular pH. However, findings in this metabolic acidosis study are consistent with ${ }^{31} \mathrm{P}$ NMR examination of the effects respiratory acidosis and alkalosis on canine myocardial $\mathrm{pH}$ in vivo (5). Unlike in vivo $\mathrm{pH}$ electrode studies, the ${ }^{31} \mathrm{P}$ NMR studies are consistent with in vitro studies $(18,21)$ which have shown that the cell is capable of maintaining $\mathrm{pH}$, despite large changes in extracellular $\mathrm{pH}$.

This buffering capability is present in the newborn myocardium, as well as the mature. The hemodynamic response to extracellular acidosis in normoxic newborn sheep has been shown to vary as a function of the experimental model and level of anesthesia $(22,23)$. This study indicates that it is unlikely for these changes in myocardial performance to be directly due to intracellular acidosis. However, during extracellular acidosis a change does occur in the hydrogen ion concentration gradient across the cell membrane. Myocardial 
contractility could conceivably be influenced by any resulting alterations in membrane electrochemical potential and ion fluxes.

In summary, this study demonstrates that significant changes occur in myocardial intracellular monophosphate and phosphomonoester concentrations during development. However, both the newborn and mature myocardium in vivo similarly demonstrate a tremendous buffering capability during extracellular acidosis. This data should be considered in analyses of the treatment of myocardium during alterations in acid-base status.

\section{Acknowledgments}

We wish to thank Drs. Ron Meyer and M. J. Fisher for reviewing this manuscript.

This work was funded, in part, by a grant from the American Heart Association of Michigan.

\section{References}

1. Portman, M. A., F. W. Heineman, and R. S. Balaban. 1989. Developmental changes in the relation between phosphate metabolites and oxygen consumption in the sheep heart in vivo. J. Clin. Invest. 83:456-464.

2. Katz, L. A., J. A. Swain, M. A. Portman, and R. S. Balaban. 1989. Relation between phosphate metabolites and oxygen consumption of heart in vivo. Am. J. Physiol. 256:H265-H274.

3. Balaban, R. S., H. L. Kantor, L. A. Katz, and R. W. Briggs. 1986. Relation between work and phosphate metabolites in the in vivo paced mammalian heart. Science (Wash. DC). 232:1121-1123.

4. Heineman, F. W., and R. S. Balaban. 1990. 31-Phosphorus NMR analysis of transient changes of canine myocardial metabolism in vivo. J. Clin. Invest. 85:843-852.

5. Katz, L. A., J. A. Swain, M. A. Portman, and R. S. Balaban. 1988. Intracellular $\mathrm{pH}$ and inorganic phosphate content of the heart in vivo. Am. J. Physiol. 255:H189-H196.

6. Balaban, R. S. 1984. The application of nuclear magnetic resonance to the study of cellular physiology. Am. J. Physiol. 246:C10C19.

7. Garlick, P. B., G. K. Radda, and P. J. Seely. 1979. Studies of acidosis in the ischemic heart by phosphorus nuclear magnetic resonance. Biochem. J. 184:547-554.

8. Pieper, G. M., G. L. Todd, S. T. Wu, J. M. Salhany, F. C. Clayton, and R. S. Sliot. 1981. Attenuation of myocardial acidosis by propanolol during ischemic arrest and reperfusion: evidence with ${ }^{31} \mathrm{P}$ nuclear magnetic resonance. Cardiovasc. Res. 14:646-653.
9. Portman, M. A., F. W. Heineman, S. James, and R. S. Balaban. 1988. Simultaneous monitoring of coronary blood flow and ${ }^{31} \mathrm{P}$ NMR detected myocardial metabolites. Magn. Reson. Med. 7:243-247.

10. Lister, G., T. K. Walter, H. T. Versmold, P. R. Dallman, and A. M. Rudolph. 1979. Oxygen delivery in lambs: cardiovascular and hematologic development. Am. J. Physiol. 237:H668-H675.

11. Brindle, K. M., B. Rajagopalan, D. S. Williams, J. A. Detre, E. Simplaceanu, C. Ho, and G. K. Radda. $1988 .{ }^{31}$ P NMR measurements of myocardial pH in vivo. Biochem. Biophys. Res. Commun. 151:7077.

12. Melby, C. E., and N. H. Altman. 1974. Handbook of Laboratory Animal Science, Vol. II. CRC Press, Inc., Cleveland, OH. 402.

13. Gyulai, L., L. Bolinger, J. S. Leigh, Jr., C. Barlow, and B. Chance. 1984. Phosphoryethanolamine-the major constituent of the phosphomonoester peak observed by ${ }^{31} \mathrm{P}$ NMR on developing dog brain. FEBS (Fed. Eur. Biochem. Soc.) Lett. 178:137-142.

14. Wolff, S. D., C. Eng, and R. S. Balaban. 1988. NMR studies of renal phosphate metabolites in vivo: effects of hydration and dehydration. Am. J. Physiol. 255(Renal Fluid Electrolyte Physiol. 24):F581F589.

15. Tofts, P., and S. Wray. 1985. Changes in brain phosphorous metabolites during the post-natal development of the rat. J. Physiol. (Lond.). 359:417-429.

16. Heerschap, A., A. H. Bergman, J. J. van Vaals, P. Wirtz, H. M. T. Loermans, and J. H. Veerkamp. 1988. Alterations in relative phosphocreatine concentrations in preclinical mouse muscular dystrophy revealed by in vivo NMR. NMR Biomed. 1:27-31.

17. B. M. Hitzig, J. W. Prichard, H. L. Kantor, W. R. Ellington, J. S. Ingwall, C. T. Burt, S. I. Helman, and J. Koutcher. 1987. NMR spectroscopy as an investigative technique in physiology. FASEB (Fed. Am. Soc. Exp. Biol.) J. 1:22-31.

18. Roos, A., and W. F. Boron. 1981. Intracellular pH. Physiol. Rev. 61:296-434.

19. Walters, F. J. M., G. J. Wilson, D. J. Steward, R. J. Domenech, and D. C. MacGregor. 1979. Intramyocardial pH as an index of myocardial metabolism during cardiac surgery. J. Thorac. Cardiovascular Surg. 78:319-330.

20. Von Planta, M., J. H. Weil, R. J. Gazmuri, J. Bisera, and E. C. Rackow. 1989. Myocardial acidosis associated with $\mathrm{CO}_{2}$ production during cardiac arrest and resuscitation. Circulation. 80:684-692.

21. Ellis, D., and R. C. Thomas. 1976. Direct measurement of the intracellular pH of mammalian cardiac muscle. J. Physiol. (Lond.). 262:755-771.

22. Fisher, D. J. 1986. Comparative effects of metabolic acidemia and hypoxemia on cardiac output and regional blood flows in unanesthetized newborn lambs. Pediatr. Res. 20:756-760.

23. Lee, J. C., K. H. Halloran, J. R. N. Taylor, and S. E. Downing. 1973. Coronary flow and myocardial metabolism in newborn lambs: effects of hypoxia and acidemia. Am. J. Physiol. 224:1381-1387. 